\title{
19 FACILITATING SUCCESSFUL IMPLEMENTATION OF THE CARER SUPPORT NEEDS ASSESSMENT TOOL (CSNAT) INTERVENTION WITHIN PALLIATIVE CARE
}

\footnotetext{
${ }^{1}$ Janet Diffin, ${ }^{2}$ Gail Ewing, ${ }^{1}$ Gunn Grande. ' University of Manchester, Manchester, UK;
} ${ }^{2}$ University of Cambridge, Cambridge, UK

10.1136/bmjspcare-2016-001204.19

Background The Carer Support Needs Assessment Tool (CSNAT) intervention identifies and addresses family carer support needs towards end of life. ${ }^{1-3}$ This person-centred approach involves a change in practice from a practitioner- to carer-led assessment process. Numerous policies recommend carers' needs should be addressed yet there is little guidance on how to achieve this within palliative care. To address this gap and assist a change to a carer-led process, an implementation strategy for the CSNAT intervention was developed, of which facilitation is key.

Aims investigate components of facilitation associated with successful implementation of the CSNAT intervention across 36 palliative care services.

Methods Qualitative: Interviews three and six months postCSNAT implementation with 38 practitioners acting as CSNAT 'champion' (internal facilitation) in 32 services. Researcher field notes collected from teleconference support sessions with 'champions' (external facilitation).

Results Successful implementation of the CSNAT intervention was associated with: a teamwork approach to facilitation; the 'lead champion' having a leadership role in the service e.g. team manager; presenting a clear rationale why a change in practice was needed; reviewing progress and making changes to overcome identified barriers; 'champions' having good communication skills and authority to make changes. Aspects of context which constrained facilitation included the need to establish a new carer record, and organisational changes e.g. budget cuts, and a culture focused on the patient. Management support assisted with facilitation efforts.

Conclusions Establishing a team of practitioners to lead facilitation of the CSNAT intervention and regularly review implementation progress, is recommended to help ensure implementation success.

\section{REFERENCES}

1 Ewing G, Brundle C, Payne S, Grande G. The Carer Support Needs Assessment Tool (CSNAT) for use in palliative and end-of-life care at home: $A$ validation study. J Pain Symptom Manage 2013;46(3):395-405

2 Ewing G, Grande G. Development of a Carer Support Needs Assessment Tool (CSNAT) for end-of-life care practice at home: A qualitative study. Palliat Med 2013:27(3):244-256

3 Ewing G, Austin L, Diffin J, Grande G. The Carer Support Needs Assessment Tool: A person centred approach to carer assessment and support. $\mathrm{Br} /$ Community Nurs 2015;20(12):580-584 